\title{
The Moving Average Filter of Stator Current as Alarm for Overload of Induction Motor
}

\author{
Goran Keković and Negovan Stamenković
}

\begin{abstract}
In this paper, the method of detecting the overload of the induction motors (IMs) by applying moving average filter of the stator current in the time domain, was suggested. This method can be analyzed as the part of the analysis of the motor current signature analysis (MCSA). The stator current is analysed as the signal and it is divided into regular intervals windows, within moving average filter applied. The minimum length of the moving window is determined by the sampling frequency detector, as well as the frequency of the stator current. The better resolution of peaks in moving average filter, can be achieved by the greater length of moving window. This is particularly important in areas of variable torque induction motor. This opens the possibility of detecting finer changes in the value of the stator current. It is shown that within the time window of changes in load of IMs, the signal of stator current becomes non-stationary. Otherwise, it is stationary. The changes of stationarity of the signal could be the alarm of overload of induction motor. The advantage of this method is improved response time of detector due to changes in the stator current. Software solution of proposed method is relatively simple: time series of the stator current is mapped according to the action of the moving average filter in different time series, suitable for use in real-time.
\end{abstract}

Index Terms-Induction Motor, Stator current, Signal analysis.

\section{INTRODUCTION}

Electric motors are now widespread and irreplaceable in various industries and production activities. Among them, the most used motors are induction motors because of properties such as robustness, reliability and durability. Therefore, special attention is paid to their maintenance and development of many techniques for timely detection of their failure. The most common failures of the IMs are bearing damage, shaft bending, stress and overload current, voltage and power underload, overload due to external mechanical torque and overheating of the stator windings. Studies show that IM failures can be classified as follows: bearing failure $40 \%$, stator failures $\sim 38 \%$, rotor failures $\sim 10 \%$ and $12 \%$ of other faults [1]. All control techniques of IMs can be divided into two categories: classical or traditional techniques and techniques based on the use of computers and microprocessors. The traditional technique involves the use of various mechanical and electrical components (relays, timers, contactors, ...), which increase the number of dynamic components of the system and reduce its efficiency. On the other hand, modern solutions based on the use of computers and microprocessors, include the conversion of analog signals into digital signals, so that ultimately the PC software decides whether to stop the engine [2], [3]. The disadvantage of these methods is that it increases the price of the cost compared to the classical technique. Control of the stator current of IMs is practically realized by the thermal relay and a microprocessor-based relay [4]. This type of relay stator winding temperature is usually determined by the thermal model of the first order. Thermal models of higher order are more accurate, but the implementation is more complicated [5], [6]. Motor Current Signature Analysis (MCSA) is analysis technique of IMs current, which considered as a signals. This method can detect some various types of damages of the motor and its overload [7], [8]. This method is based on Fourier transformation (FFT) of stator current. The appearance of multiple peaks in the power spectra of the stator current, can be an indicator of bearing damage, broken rods or motor overload. Properties of the peaks are determined by the type of damage output [9]. The main disadvantages of FFT is the fact that it is applicable only to stationary signals, and that this method gives only the distribution of power in the frequency domain, but not in the time domain. This problem is solved in the signal analysis by introducing Wavelet transformation [10], [11]. In the core of this method is the solving convolution integral at the each point of the signal. Convoluion integral is solved by applying various, so-called, "mother wavelets" and their successors at the different frequencies. The solutions of convolution integral (details) represent desired distrubution in the frequency and time domain. However, the problem of stationarity of signal still persists. In biosignal analysis, this problem is solved by dividing the signal in the stationary segments. Determination of the boundaries of these segments (change point detection - CPD), is not simple and it is associated with the introduction of a series of parameters, dependent on the type of signal. This further complicates the problem and especially its technical solution.

In this paper a simple solution of this problem is suggested and it is based on moving average filter of stator current. Namely, when an overload of IMs occurs, the stator current becomes non-stationary. In these points, changes within a moving sliding window of average values are much more pronounced than the original stator current. These changes occur before the signal of stator current reaches its maximum, indicating the possibility of shortening the response time of the detector.

Negovan Stamenković, University of Priština, Serbia. (e-mail: negovanstamenkovic@gmail.com). 


\section{MATERIAL AND METHODS}

\section{A. The mathematical model of the induction motor}

In a theoretical experiment in this paper, the influence of a variable load torque on the stator current of squirrel-cage induction motor was analysed. The following assumptions were taken into account: a uniform air gap, balanced rotor and stator windings, saturation and parameter changes are ignored. The standard system of differential equation has been solved [12], [13]:

$$
\begin{gathered}
\frac{d \Psi_{q s}}{d t}=\omega_{b}\left[V_{q s}-\left(\frac{\omega_{e}}{\omega_{b}}\right) \Psi_{d s}-\left(\frac{R_{s}}{X_{l s}}\right)\left(\Psi_{m q}-\Psi_{q s}\right)\right] \\
\frac{d \Psi_{d s}}{d t}=\omega_{b}\left[V_{d s}-\left(\frac{\omega_{e}}{\omega_{b}}\right) \Psi_{q s}-\left(\frac{R_{s}}{X_{l s}}\right)\left(\Psi_{m d}-\Psi_{m s}\right)\right] \\
\frac{d \Psi_{q r}}{d t}=\omega_{b}\left[V_{q r}-\left(\frac{\omega_{e}-\omega_{r}}{\omega_{b}}\right)-\left(\frac{R_{r}}{X_{l r}}\right)\left(\Psi_{m d}-\Psi_{d r}\right)\right] \\
\frac{d \Psi_{d r}}{d t}=\omega_{b}\left[V_{d r}-\left(\frac{\omega_{e}-\omega_{r}}{\omega_{b}}\right)-\left(\frac{R_{r}}{X_{l r}}\right)\left(\Psi_{m d}-\Psi_{d r}\right)\right] \\
\frac{d \omega_{b}}{d t}=\frac{p}{2 J}\left[T_{e}-T_{l}\right]
\end{gathered}
$$

where are $T_{e}, T_{l}$, electromagnetic and mechanical load torque, $p$ is the number of poles, and $J$ is the moment of inertia of the motor axis. Corresponding fluxes in the two-dimensional Q, D system are given as:

$$
\begin{gathered}
\Psi_{m q}=X_{m l}\left\lceil\frac{\Psi_{q s}}{X_{l s}}+\frac{\Psi_{q r}}{X_{l r}}\right\rceil \\
\Psi_{m d}=X_{m l}\left\lceil\frac{\Psi_{d s}}{X_{l s}}+\frac{\Psi_{d r}}{X_{l r}}\right\rceil .
\end{gathered}
$$

The corresponding equations linking fluxes with currents are:

$$
\begin{aligned}
& i_{q s}=\frac{1}{X_{l s}}\left[\psi_{q s}-\psi_{m q}\right] \\
& i_{q r}=\frac{1}{X_{l r}}\left[\psi_{q r}-\psi_{m q}\right] \\
& i_{d s}=\frac{1}{X_{l r}}\left[\psi_{d s}-\psi_{m d}\right] \\
& i_{d r}=\frac{1}{X_{l r}}\left[\psi_{d r}-\psi_{m d}\right]
\end{aligned}
$$

Electromagnetic torque $T_{e}$ and the quantity are described by expressions:

$$
\begin{aligned}
& T_{e}=\frac{3 p}{4 J \omega_{b}}\left[\Psi_{d s} i_{q s}-\Psi_{q s} i_{d s}\right] \\
& X_{m l}=\frac{1}{\frac{1}{X_{m}}+\frac{1}{X_{l s}}+\frac{1}{X_{l r}}}
\end{aligned}
$$

The corelation between the currents $i_{d}, i_{q}$ in two dimensional system, current stator and rotor currents $i_{\alpha}, i_{\beta}$ and line currents $i_{a}, i_{b}, i_{c}$ are given as:

$$
\left[\begin{array}{l}
i_{\alpha} \\
i_{\beta}
\end{array}\right]=\left|\begin{array}{cc}
\cos \Theta & -\sin \Theta \\
\sin \Theta & \cos \Theta
\end{array}\right|\left[\begin{array}{l}
i_{d} \\
i_{q}
\end{array}\right]
$$

$$
\begin{gathered}
{\left[\begin{array}{l}
i_{a} \\
i_{b} \\
i_{c}
\end{array}\right]=\frac{2}{3}\left[\begin{array}{cc}
1 & 0 \\
-\frac{1}{2} & -\frac{\sqrt{3}}{2} \\
-\frac{1}{2} & -\frac{\sqrt{3}}{2}
\end{array} \mid\left[\begin{array}{l}
i_{\alpha} \\
i_{\beta}
\end{array}\right]\right.} \\
\Theta=\omega_{e} t
\end{gathered}
$$

In this numerical simulation three-phase balanced system was used:

$$
\begin{aligned}
& V_{a}=\sqrt{2} V_{r s m} \sin (\omega t) \\
& V_{b}=\sqrt{2} V_{r s m} \sin \left(\omega t-\frac{2 \pi}{3}\right) \\
& V_{c}=\sqrt{2} V_{r s m} \sin \left(\omega t+\frac{2 \pi}{3}\right)
\end{aligned}
$$

where: $V_{a}=V_{b}=V_{c}=400 \mathrm{~V}, f=50 \mathrm{~Hz}$. The properties of the IMs: $P=4 \mathrm{~kW}, L_{s}=L_{r}=5,84 \mathrm{mH}, L_{m}=172,2 \mathrm{mH}, L_{s m}=L_{r m}=$ $166,36 \mathrm{mH}, n_{r}=1430 \frac{\mathrm{ob}}{\mathrm{min}}, J=0,0131 \frac{\mathrm{kg}}{\mathrm{m}^{2}}, p=2$. Mechanical torque of external load is controlled as follows:

$$
T_{L}=\left\{\begin{array}{cc}
0 ; & 0<t \leq 1 s \\
50 \mathrm{Nm} ; & 1 s<t \leq 2 s \\
120 \mathrm{Nm} ; & 2 s<t \leq 3 s
\end{array}\right\}
$$

Iteration simulation step was $\Delta t=10^{-5} \mathrm{~s}$, while the total duration was $t=3 \mathrm{~s}$. In the next section, the algorithm of moving average filter was proposed as a solution for detecting a variable load torque of IMs.

\section{B. Algorithm of moving average filter}

The stator current is analysed as a time series and its changes are determined as follows:

1. Select the length of the time window. Let the sampling frequency of detector $f_{s}$ and frequency of stator current $f$. Then, the length of sliding window should be:

$$
L=\frac{T}{T_{s}}=\frac{f_{s}}{f}
$$

In the case of stationary signal, $L=$ const. This condition is fulfilled in normal working conditions of IMs, when stator current has its nominal values. At the points of changes of loads of IMs, the frequency $f$ of signal (stator current) changes and signal becomes non-stationary. That leads to variable length of time windows, $L \neq$ const and sharp peaks in spectra of moving average filter occur. The linear relation between $f, L$ does not hold anymore and it is very difficult to find analytical expression of that relation. After numerical experimentations, the optimum length of time sliding window was found. It was 4000 samples or $0,04 \mathrm{~s}$. Within this window, changes in load of IMs had most pronounced peaks in moving average filter values.

2. Divide signal into overlapping sliding windows of length of $L$, centered in index $\mathrm{i}$ and find average 
value:

$$
y_{i}=\frac{1}{L} \sum_{k=i-\frac{L}{2}}^{i+\frac{L}{2}} x_{k}
$$

For use in real-time it can be easily shown that following recurrent formula holds:

$$
y_{i+1}=y_{i}+\frac{1}{L} \cdot\left(x_{i+1+\frac{L}{2}}-x_{i-\frac{L}{2}}\right)
$$

3. Set threshold of determining of peaks in response of moving average filter. Since, the signal of stator current is a harmonic function: $x \sim x_{0} \sin \omega t$, from equation (9) follows that new signal $y_{i}$ has the properties of harmonic function. From equation (10) it follows:

$$
y_{i+1}-y_{i}=\frac{1}{L} \cdot\left(x_{i+1+\frac{L}{2}}-x_{i-\frac{L}{2}}\right) \leq \frac{2 \Delta x_{k r}}{L}=2 y_{k r}
$$

where $x_{k r}, y_{k r}$ represents the critical values of overload of IMs. Since the threshold of overload is usually $125 \%$ of stator current under maximum overload, from (10) it follows: $2 y_{k r}=2$. $1,25 y_{\max }=2,5 y_{\max }$. The selected threshold was: 2,9 $9 y_{\max }$, within $T=50 \mathrm{Nm}$, so the points $y_{i}$ above and below threshold are expected. The benefit of this approach is that violation of threshold starts before the signal of stator current reaches its values characteristical for overloads of IMs.

\section{RESULTS AND DISCUSSION}

The value of torque of the load, at which the engine speed drops to zero was $T=120 \mathrm{Nm}$. In Fig. 1a the stator current is shown, with corresponding changes in load of IMs, marked by dashed lines. The response of moving average filter of the signal in time domain is shown in Fig. 1b. The changes in load of IMs are emphasized as a peak in this response. In Fig. 2 these changes are zoomed, for load of $T=50 \mathrm{Nm}$, Fig. 2a (signal), Fig. 2c (filter) and for overload of $T=120 \mathrm{Nm}$, Fig. 2b (signal) and Fig. 2d (filter). It can be clearly seen that the trip below the threshold in Fig. $2 d$ (dashed line, denoted by $t_{\text {avg }}$ ) prior to the onset values of the stator current of overload of IMs (dashed line, $t_{\text {det }}$ ). This phenomenon allows earlier detection of changes in load of IMs. The signal can be considered as stationary if its central tendency (mean, median and standard deviation) is constant in time [14], [15]. Another important indicator of its stationarity is autocorrelation function. If it slowly drops to zero the signal can be regarded as stationary, otherwise, it is non-stationary [16]. Also, violation of stationarity signal during changing of motor load can be visually observed on the basis of zero crossings in signal $y$. This suggests that in the frequency spectrum of the stator current additional peaks may occur. This is consistent with the results of the research of signature of overload in the stator current [17]. On the other hand, there is (Fig. 1a) constant distance between the zeroes of the rotor current in steady conditions of IMs. In contrast, this distance is not constant under non-steady conditions (time before pull-up

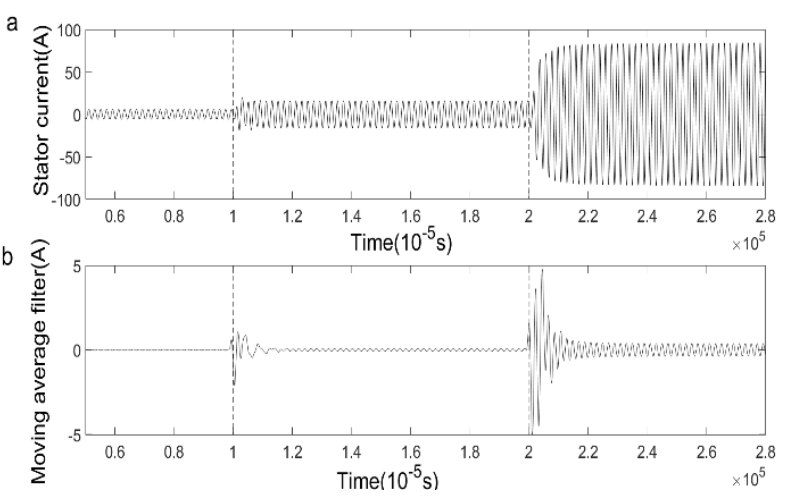

Fig. 1. The stator current of the induction motor at varying engine load of $T_{L}=0-120 \mathrm{Nm}$; (a) the signal of the stator current $i_{s} ;(\mathrm{b})$ the signal of the moving average filter $i_{a v}$.
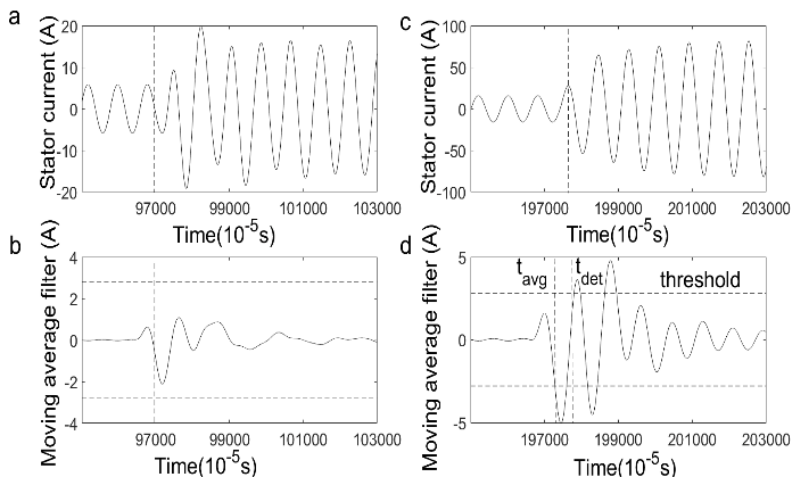

Fig. 2. Stator currents during changes of the load of IMs, $T_{L}=0-50 \mathrm{Nm}$ (a) and $T_{L}=50 \mathrm{Nm}-120 \mathrm{Nm}$ (b) as well corresponding moving average filters for $T_{L}=0-$ $50 \mathrm{Nm}$ (c) and for $T_{L}=50 \mathrm{Nm}-120 \mathrm{Nm}$ (d).

torque). This points to the fact that the signal in that part, nonstationary. That part was not considered in this work. The moment of occurrence of the critical load is determined on the basis of the above mentioned algorithm. According to the equation (7) the critical change in load of IMs occurs at the time $t=2 s$, while in spectra of moving average filter appeared, $\Delta t=\frac{L}{2}=0.02 \mathrm{~s}$ earlier. The next logical question is whether this method can distinguish between failures and overload of induction motor. In a wide literature electrical and mechanical failures, as well as overloads of IMs are described and they leave characteristic signatures of the stator current [18], [8]. In the power spectrum characteristic peak occurs at the center frequency $\left(f_{e}=60 \mathrm{~Hz}\right.$, in our case) and accompanying peaks around it. Most of these methods are based on the application of Fourier transform, which is not applicable in the cases of non-stationary signals. In the analysis of the biosignals, this problem is solved by detecting the change point in the stationarity of the signal (CPD). For this purpose, numerous methods have been proposed [19], [20]. The essence of these methods is the detection of the change points of stationarity of a signal, whereupon the FFT to the thus defined stationary intervals of signal is applied. Determination of the CPD could be achieved with filter of moving average value, as well as with the wavelet filter [21]. There is the question of technical implementation of detector 
based on the method proposed in this work. The proposed filter can be used in conjunction with superfast solid state relays, whose typical response time is of order $\sim 100 \mu$ s. On the other hand, the fastest time of classical thermal relay is $5 \mathrm{~s}$ and the proposed filter can not be used in this case. Trip time of the relays is determined on the basis of the thermal curves, which depend on type of IMs and its vendor [22]. Another possible solution is counting zeroes of stator current. There are various technical solutions for this, mainly based on comparators and optocouplers [23], [24]. The focus is on the theoretical analysis of the proposed method, so we will not go into hardware details.

\section{REFERENCES}

[1] C. J. Verucchi, G. G. Acosta, and F. A. Benger, "A Review On Fault Diagnosis Of Induction Machines," Latin American Applied Research, vol. 38, no. 5, pp. 113-121, 2008.

[2] I. Colak, H. Celik, I. Sefa, and S. Demirbas, "On line protection system for induction motors," Energy Conversion and Management, vol. 46, no. 17, pp. 2773-2786, March 2005.

[3] M. G. Ioannides, "Design and Implementation of PLC-Based Monitoring Control System for Induction Motor," IEEE Transactions On Energy Conversion, vol. 19, no. 3, pp. 469 - 476, October 2004.

[4] A. Gedzurs, "Induction Motor Stator Winding Thermal Process Research and Modelling under Locked Rotor Mode," Proceedings of 14th International Scientific Conference. Engineering for Rural Development, pp. 361 - 365, May 2015.

[5] J. F. Moreno, F. P. Hidalgo, M. D. Martinez, "Realisation of tests to determine the parameters of the thermal model of an induction machine," Electric Power Applications, IEE Proceedings, vol. 148, pp. 393-397, September 2001.

[6] P. Zhang, B. Lu, T.G. Habetler, "Active stator winding thermal protection for AC motors," presented at Pulp and Paper Industry Technical Conference, Birmingham, 21-26 June, 2009.

[7] G.B. Kliman, R.A. Koegl, J. Stein, R.D. Endicott et M.W. Madden, "Noninvasive detection of broken rotor bars in operating induction motors," IEEE Trans. Energy Conv., vol. EC3, no. 4, pp. 873-879, December 1988

[8] T. Gökta, M. Arkan, Ö. F. Özgüven "Detection of rotor fault in threephase induction motor in case of low-frequency load oscillation," Electric Eng., vol. 97, pp. 337-345, July 2015.

[9] A. Vatsa, "A brief review of condition monitoring practices of induction motor," International Journal of Research and Engineering, vol 4. no. 3, 86 - 92, March 2017.

[10] A. Metin, Time-frequency and wavelets in biomedical signal processing, IEEE Computer Society Press, USA, 1997, pp. 211-222.

[11] S. G. Khalaf, and Hew W. P. Hew, "Wavelet Fault Diagnosis of Induction Motor," MATLAB for Engineers - Applications in Control, Electrical Engineering, IT and Robotics, 341-364, Karel Perutka, K. IntechOpen, 2011, pp. 341-364.

[12] P.C. Krause, and C. H. Thomas, "Simulation of Symmetrical Induction Machinery," IEEE Transaction on Power Apparatus and Systems, vol. 84, pp. 1038-1053, November 1965.

[13] P. L. Ratnanil, and A. G. Thosar, "Mathematical Modelling of an 3 Phase Induction Motor Using MATLAB/Simulink," International Journal Of Modern Engineering Research, vol. 4, no. 6, pp. 62 - 67, June 2014.

[14] R. Cédric, A. Ferrari, H. Amoud, P. Honeine, P. Flandrin, and P. Borgnat, "Statistical hypothesis testing with time-frequency surrogates to check signal stationarity," presented at IEEE International Conference on Acoustics, Speech, and Signal Processing ICASSP-10, Dallas, Texas, pp. 3666-3669, March 14-19, 2010.

[15] X. Jun, P. Borgnat, P. Flandrin, C. Richard, "Testing Stationarity with Surrogates - A One-Class SVM Approach," IEEE/SP 14th Statistical Signal Processing Workshop (SSP '07), pp. 720-724, August 2007.

[16] F. By, L. Ramsey, "Characterization Of The Partial Autocorrelation Function," The Annals of Statistics, 2(6), pp. 1296 - 1301, November 1974.

[17] P. Pillay, and Xu. Z, "Motor Current Signature Analysis," presented at Thirty-First IAS Annual Meeting, Industry Applications Conference. '96, pp. 587-594, October, 1996.

[18] 18. M. Zeraoulia, A. Mamoune, H. Mangel, and M. E. H. A. Benbouzid, "Simple Fuzzy Logic Approach for Induction Motors

\section{CONCLUSION}

In this article the method of determining of overload of IM was analysing, by applying moving average filter to the stator current. It is shown that in case of the overload of IM, the signal of stator current becomes non-stationary. This method is free of shortcomings of spectral analysis current in terms of signal stationarity. It is simple in terms of software implementation. It is necessary to analyse the application of this method in cases of various defects of IMs. This is left for future work.

Stator Condition Monitoring," J. Electrical Systems, vol. 1, no. 1, pp. $15-25,2005$

[19] S. M. Hammoudeh, and H. LI, "Sudden changes in volatility in emerging markets: The case of Gulf Arab stock markets," International Review of Financial Analysis, vol. 17, no. 1, pp. 47-63, 2008.

[20] P. Yang, G. Dumont, J.M. Ansermino, "Adaptive change detection in heart rate trend monitoring in anesthetized children," IEEE Trans Biomed Eng, vol. 53, no. 11, pp. 2211-2219, 2006.

[21] G. Keković, and S. Sekulić, "Detection of Change Points in Time Series with Moving Average Filters and Wavelet Transform: Application to EEG Signals," Neurophysiology, vol. 1, no. 51, pp. 29, May 2019.

[22] J. Steinmetz, C. P. Subhash, and S. E. Zocholl, "Stator thermal time constant," presented at 49th IEEE/IAS Industrial \& Commercial Power Systems Technical Conference, pp. 1-7, April 30-May 3, 2013.

[23] http://www.silego.com/uploads/Products/product_677.

[24] Optocoupler, Phototriac Output, Zero Crossing, High dV/dt, Low Input Current, Vishay Semiconductors, IL410, IL4108, https://www.vishay.com/docs/83627/i1410.pdf. 